\title{
New Bloch period for interacting cold atoms in 1D optical lattices
}

\author{
Andrey R. Kolovsky \\ Max-Planck-Institut für Physik Komplexer Systeme, D-01187 Dresden, Germany and \\ Kirensky Institute of Physics, 660036 Krasnoyarsk, Russia
}

(Dated: October 29, 2018)

\begin{abstract}
This paper studies Bloch oscillations of ultracold atoms in an optical lattice, in the presence of atom-atom interactions. A new, interaction-induced Bloch period is identified. Analytical results are corroborated by realistic numerical calculations.

PACS numbers: PACS: 32.80.Pj, 03.65.-w, 03.75.Nt, 71.35.Lk
\end{abstract}

The response of a quantum system to a static field has been a longstanding problem since the early days of quantum mechanics. A topic of particular interest in this wide field is the dynamics of a quantum particle in a periodic potential induced by a static force (modelling a crystal electron in an electric field). In this system, the effect of the field manifests in a very unintuitive way. Indeed, as already emphasised by Bloch [1] and Zener [2], according to the predictions of wave mechanics, the motion of electrons in a perfect crystal should be oscillatory rather than uniform. This phenomenon, nowadays known as Bloch oscillations (BO), has recently received renewed interest which was stimulated by experiments on cold atoms in optical lattices [3, 4, 5, 6]. This system (which mimics a solid state system - with the electrons and the crystal lattice substituted by the neutral atoms and the optical potential, respectively) offers unique possibilities for the experimental study of $\mathrm{BO}$ and of related phenomena. In turn, these fundamentally new experiments have stimulated considerable progress in theory (see review 7], and references therein), and it can be safely stated that $\mathrm{BO}$ in diluted quasi one-dimensional gases is well understood today. Other directions of research focus on $\mathrm{BO}$ in the presence of relaxation processes (spontaneous emission) [8], $\mathrm{BO}$ in 2D optical lattices [9], and $\mathrm{BO}$ in the presence of atom-atom interactions ('BEC-regime') 10, 11, 12, 13. The present Letter deals with the third problem, which is approached here by an 'ab initio' analysis of the dynamics of a system of many atoms. This distinguishes this work from previous studies of $\mathrm{BO}$ in the $\mathrm{BEC}$ regime 10, 11, 12], which were based on the a mean field approach using a nonlinear Schrödinger equation. A new effect, so far unaddressed by these earlier studies, is predicted: besides the usual Bloch dynamics, the atomic oscillations may exhibit another fundamental period, entirely defined by the strength of the atom-atom interactions.

Let us first recall some results on $\mathrm{BO}$ in the singleparticle case. Using the tight-binding approximation [14], the Hamiltonian of a single atom in an optical lattice has the form

$$
H=E_{0} \sum_{l}|l\rangle\langle l|-\frac{J}{2}\left(\sum_{l}|l+1\rangle\langle l|+h . c .\right)
$$

$$
+d F \sum_{l} l|l\rangle\langle l|
$$

In Eq. (11), $|l\rangle$ denotes the $l$ th Wannier state $\phi_{l}(x)$ corresponding to the energy level $E_{0}[15], J$ is the hopping matrix elements between neighbouring Wannier states, $d$ is the lattice period, and $F$ is the magnitude of the static force. The Hamiltonian (11) can be easily diagonalised, which yields the spectrum $E_{l}=E_{0}+d F l$ (the so-called Wannier-Stark ladder) and the eigenstates (Wannier-Stark states)

$$
\left|\psi_{l}\right\rangle=\sum_{m} \mathcal{J}_{m-l}(J / d F)|m\rangle, \quad\langle x \mid m\rangle=\phi_{m}(x)
$$

(here $\mathcal{J}_{m}(z)$ are the Bessel functions). As a direct consequence of the equidistant spectrum, the evolution of an arbitrary initial wave function is periodic in time, with the Bloch period $T_{B}=2 \pi \hbar / d F$. In particular, we shall be interested in the time evolution of the Bloch states $\left|\psi_{\kappa}\right\rangle=\sum_{l} \exp (i d \kappa l)|l\rangle$. Using the explicite expression for the Wannier-Stark states (2), it is easy to show that $\left|\psi_{\kappa}(t)\right\rangle=\exp \{-i(J / d F) \sin (d \kappa(t))\}\left|\psi_{\kappa(t)}\right\rangle$, where $\kappa(t)=\kappa+F t / \hbar$ (from now on $E_{0}=0$ for simplicity). Note that the exponential pre-factor in the last equation contains the same parameter $J / d F$ as the argument of the Bessel function in Eq. (2). Depending on the value of this parameter, the regimes of weak $(d F \ll J)$ or strong $(d F \gg J)$ static fields can be distinguished. In this Letter, we shall restrict ourselves to the strong field case, which, in some sense, is easier to treat than the weak field regime. Indeed, for $J / d F \ll 1$, the WannierStark states practically coincide with Wannier states, and $\left|\psi_{\kappa}(t)\right\rangle \approx\left|\psi_{\kappa(t)}\right\rangle$.

A remark concerning the characteristic values of the parameters is at place here: In the numerical simulations below, we use scaled variables, where $\hbar=1, d=2 \pi$, and the energy is measured in units of the photon recoil energy. In typical experiments with cold atoms in an optical lattice, the amplitude $v$ of the optical potential equals few recoil energies. Then, for example, for $v$ equal to 10 recoil energies, the value of the dimensionless hopping matrix element is $J=0.0384$. The strength of the static field is restricted from below by the condition $d F>J$, and from above by the condition that Landau-Zener tunnelling events can be neglected. 


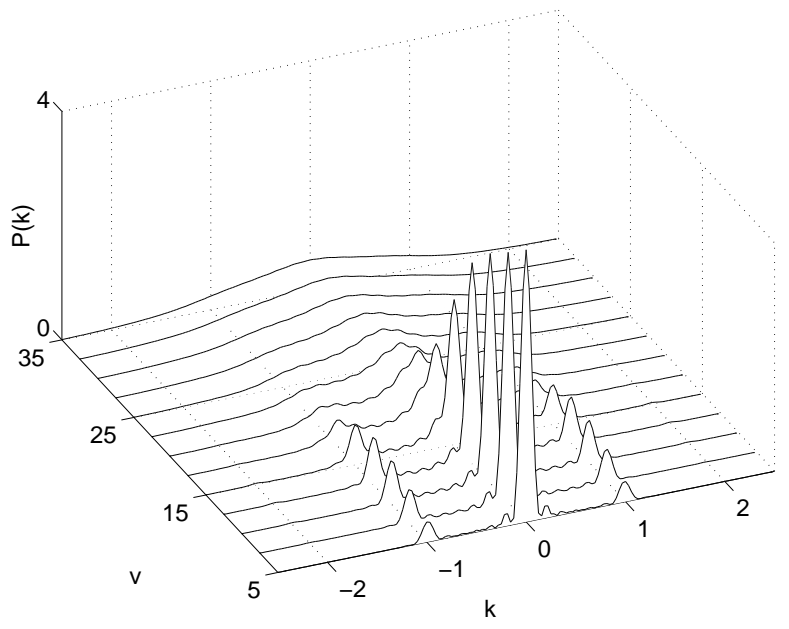

FIG. 1: Momentum distribution of the atoms in the optical lattice, for different amplitudes $v$ of the optical potential. (The amplitude $v$ is measured in units of the recoil energy, the momentum $k$ in units of $2 \pi \hbar / d$.) The figure illustrates the transition from the SF-phase to the MI-phase as $v$ is varied $(F=0, L=N=7)$.

Since the probability of Landau-Zener tunnelling is proportional to $\exp \left(-\pi \delta^{2} / 8 d F J\right)$ ( $\delta$ is the energy gap separating the lowest Bloch band from the remaining part of the spectrum) [2, 7], we have $F<30$ for $v=10$.

We proceed with the multi-particle case. A natural extension of the tight-binding model (1), which accounts for the repulsive interaction of the atoms, is given by the Bose-Hubbard model [16],

$$
\begin{gathered}
H=-\frac{J}{2}\left(\sum_{l=1}^{L} \hat{a}_{l+1}^{\dagger} \hat{a}_{l}+\text { h.c. }\right)+\frac{W}{2} \sum_{l=1}^{L} \hat{n}_{l}\left(\hat{n}_{l}-1\right) \\
+2 \pi F \sum_{l=1}^{L} l \hat{n}_{l}
\end{gathered}
$$

In Eq. (3), $\hat{a}_{l}^{\dagger}$ and $\hat{a}_{l}$ are the bosonic creation and annihilation operators, $\hat{n}_{l}=\hat{a}_{l}^{\dagger} \hat{a}_{l}$ is the occupation number operator of the $l$ th lattice site, and the parameter $W$ is proportional to the integral over the Wannier function raised to the fourth power. Since the Bose-Hubbard Hamiltonian conserves the total number of atoms $N$, the wave function of the system can be represented in the form $|\Psi\rangle=\sum_{\mathbf{n}} c_{\mathbf{n}}|\mathbf{n}\rangle$, where the vector $\mathbf{n}$, consisting of $L$ integer numbers $n_{l}\left(\sum_{l} n_{l}=N\right)$, labels the $N$-particle bosonic wave function constructed from $N$ Wannier functions. (In what follows, if not stated otherwise, $|\Psi\rangle$ refers to the ground state of the system.) As known, in the thermodynamic limit, and for $F=0$, the system (3) shows a quantum phase transition from a superfluid (SF) to a Mott insulator (MI) phase as the ratio $J / W$ is varied (see [17] and references therein). It is interesting to note that an indication of this transition can already be observed

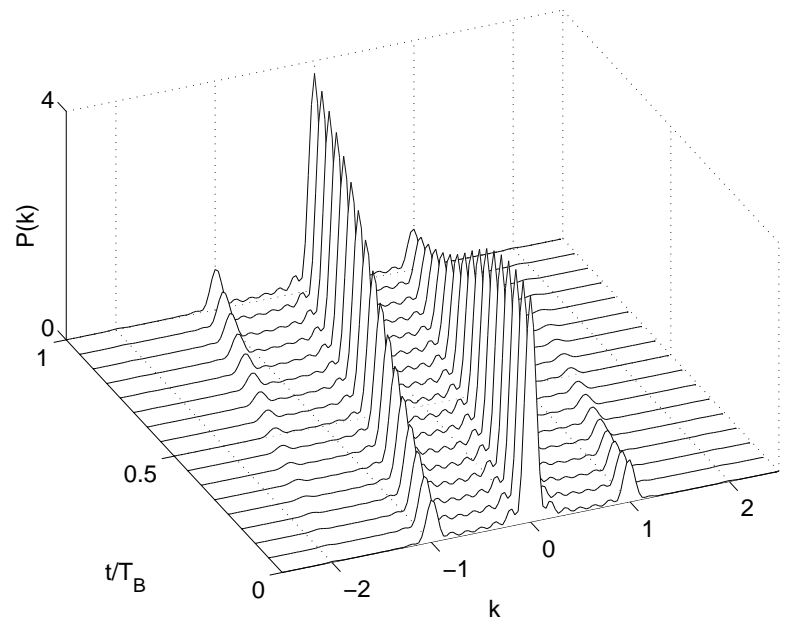

FIG. 2: Bloch oscillations of the atoms, induced by the static force $F=1 / 2 \pi(v=10)$. One Bloch period is shown.

in a system of few atoms [18]. As an example, Fig. 1 shows the diagonal elements of the one-particle density matrix,

$$
\rho\left(k, k^{\prime}\right)=\left\langle\Psi\left|\hat{\Phi}^{\dagger}(k) \hat{\Phi}\left(k^{\prime}\right)\right| \Psi\right\rangle, \quad \hat{\Phi}(k)=\sum_{l=1}^{L} \hat{a}_{l} \phi_{l}(k),
$$

for $N=L=7,5 \leq v \leq 35$, and $W=0.1 \int d k \phi_{l}^{4}(k)$ (here, $\phi_{l}(k)$ are the Wannier states in the momentum representation, and $k=p /(2 \pi \hbar / d)$ is the dimensionless momentum). Physically, this quantity corresponds to the momentum distribution $P(k)=\rho(k, k)$ of the atoms, directly measured in the experiment. It is seen in Fig. 1 that, around $v=15$, there is a qualitative change in the momentum distribution, in close analogy with that observed in the experiment [19]. It should be noted, however, that this qualitative change of the momentum distribution alone does not yet prove the occurrence of a phase transition. A more reliable indication of a SF-MI phase transition are the fluctuations of the number of atoms in a single well, which drops from $\left\langle\Delta n^{2}\right\rangle \approx 0.72$ at $v=5$ to $\left\langle\Delta n^{2}\right\rangle \approx 0$ at $v=35[20]$.

Let us now discuss the effect of the static force. Figure 2 shows the dynamics of the momentum distribution of the atoms (which were initially in SF-phase) in presence of a force $F=1 / 2 \pi$ [21]. This numerical simulation illustrates atomic $\mathrm{BO}$ as observed in laboratory experiments [3, 13. It is seen that after one Bloch period the initial momentum distribution practically coincides with the final distribution. A small difference, which can be noticed by closer inspection of the figure, is obviously due to the atom-atom interaction 22]. This difference becomes evident once the system evolved over several Bloch periods. In Fig. 3] the momentum distribution $P(k)$ at integer multiples of the Bloch period is shown. A periodic change of the distribution from SF to MI-like and back is clearly seen. (The use of the term 'MI-like' stresses the fact that the variance $\left\langle\Delta n^{2}\right\rangle$ does not change as time evolves.) In 


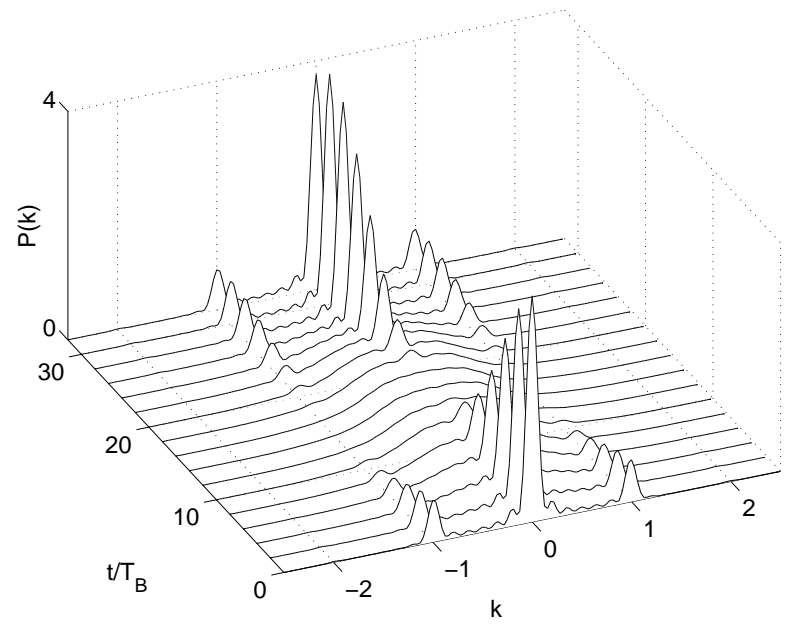

FIG. 3: Dephasing of Bloch oscillations due to the atomatom interaction. The period $T_{W}=2 \pi F / W$ is clearly seen. $\left(F=1 / 2 \pi, v=10, W=0.1 \int d x \phi_{l}^{4}(x)=0.0324\right.$. $)$
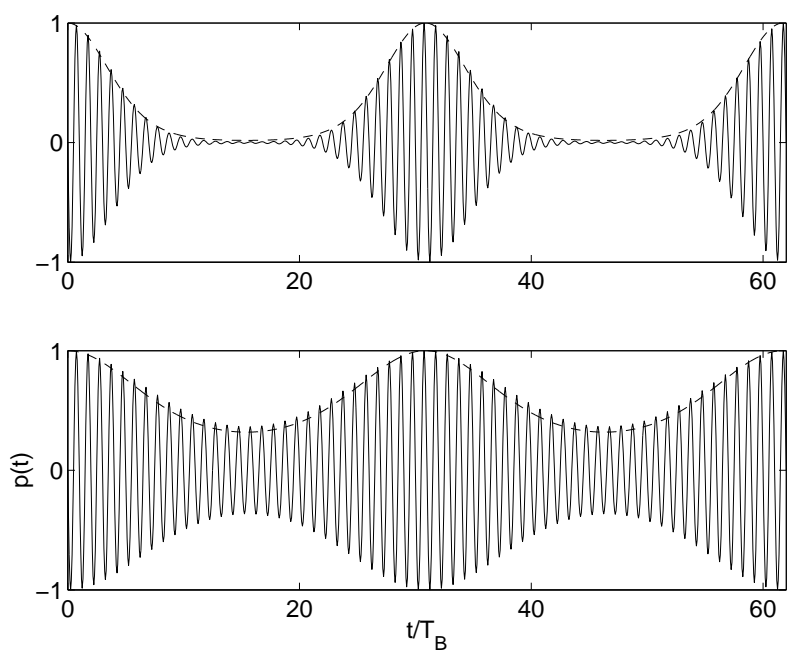

FIG. 4: Normalised mean momentum $(p / N J \rightarrow p)$ as a function of time, for two different values of the occupation number $\bar{n}=N / L: \quad N=L=7$ (upper panel), and $N=4, L=14$ (lower panel). The dashed lines show the analytical result for the envelope function in the thermodynamic limit.

addition to Fig. 2 and Fig. 3 Fig. 4 depicts the mean momentum $p(t)$ of the atoms for two different values of the occupation number $\bar{n}=N / L$ (number of atoms per lattice cite) $-\bar{n}=1$ (upper panel) and $\bar{n}=2 / 7$ (lower panel). As to be expected, the dynamics of the system depends on the value of $\bar{n}$, and for a larger occupation number the deviations of many-particle BO from the noninteracting result $p(t)=N J \sin (2 \pi F t)$ becomes larger.

Our explanation for the numerical results is the following. It is convenient to treat the atom-atom interaction as a perturbation. Let us denote by $U_{0}(t)$ the evolution operator of the system for $W=0$, by $U(t)$ the evolution operator for $W \neq 0$, and by $U_{W}(t)$ the evolution operator defined by the decomposition $U(t)=U_{W}(t) U_{0}(t)$. Since
$U_{0}\left(T_{B}\right)$ is the identity matrix, one has to find $U_{W}\left(T_{B}\right)$ to reproduce the result of Fig. [3 In the interaction representation, the formal solution for $U_{W}\left(T_{B}\right)$ has the form

$U_{W}\left(T_{B}\right)=\widehat{\exp }\left(-i \frac{W}{2} \int_{0}^{T_{B}} d t U_{0}^{\dagger}(t) \sum_{l=1}^{L} \hat{n}_{l}\left(\hat{n}_{l}-1\right) U_{0}(t)\right)$,

where the hat over the exponential denotes time ordering. Now we make use of the above strong-field condition $d F>J$. Under this premise the Wannier states are the eigenstates of the atom in the optical lattice [see Eq. (2)]. In the multi-particle case this means that the Fock states $|\mathbf{n}\rangle$ are the eigenstates of the system (3) and, thus, that the operator $U_{0}(t)$ is diagonal in the Fock state basis. Then the integral in Eq. (5) can be calculated explicitely, which yields

$$
\left\langle\mathbf{n}\left|U_{W}\left(T_{B}\right)\right| \mathbf{n}\right\rangle=\exp \left(-i \frac{W}{2 F} \sum_{l=1}^{L}\left\langle\mathbf{n}\left|\hat{n}_{l}\left(\hat{n}_{l}-1\right)\right| \mathbf{n}\right\rangle\right) .
$$

Finally, by noting that the quantity $\left\langle\mathbf{n}\left|\hat{n}_{l}\left(\hat{n}_{l}-1\right)\right| \mathbf{n}\right\rangle$ is always an even integer, one comes to the conclusion that, besides the Bloch period, there is additional period,

$$
T_{W}=2 \pi F / W
$$

which characterises the dynamics of the system.

Further analytical results can be obtained if we approximate the ground state of the system for $F=0$ by the product of $N$ Bloch waves with quasimomentum $\kappa=0$, i.e.,

$$
|\Psi\rangle=\frac{1}{\sqrt{N !}}\left(\frac{1}{\sqrt{L}} \sum_{l=1}^{L} \hat{a}_{l}^{\dagger}\right)^{N}|0 \ldots 0\rangle .
$$

Indeed, let us consider, for example, the dynamics of the mean momentum. Using the interaction representation (now with respect to the Stark energy term) the mean momentum is given by

$$
p(t)=J \operatorname{Im}\left(\left\langle\Psi U_{W}^{\dagger}(t)\left|\sum_{l=1}^{L} \hat{a}_{l+1}^{\dagger} \hat{a}_{l}\right| U_{W}(t) \Psi\right\rangle e^{-i 2 \pi F t}\right),
$$

where $U_{W}(t)$ is the continuous-time version $\left(T_{B} \rightarrow t\right)$ of the diagonal unitary matrix (6). Substituting Eq. (8) and Eq. (6) into Eq. (9), we obtain the following exact expression,

$$
\frac{p(t)}{N J}=\frac{L}{N} \operatorname{Im}\left(\sum_{n, n^{\prime}} n \mathcal{P}\left(n, n^{\prime}\right) e^{i\left(n^{\prime}-n+1\right) W t} e^{-i 2 \pi F t}\right),
$$

where $\mathcal{P}\left(n, n^{\prime}\right)$ is the joint probability to find $n$ and $n^{\prime}$ atoms in two neighbouring wells. In the thermodynamic limit $N, L \rightarrow \infty, N / L=\bar{n}$, the function $\mathcal{P}\left(n, n^{\prime}\right)$ factorises into a product of the Poisson distributions $\mathcal{P}(n)=\bar{n}^{n} \exp (-\bar{n}) / n$ !, and the double sum 
in Eq. (10) converges to the positive periodic function, $f(t)=\exp (-2 \bar{n}[1-\cos (W t)])$, indicated in Fig. 4 by the dashed line. Good agreement between the envelope of $p(t)$ and the dashed line proves that in the numerical simulation presented above the convergence was indeed achieved.

In summary, Bloch oscillations of interacting cold atoms have been studied, both numerically and analytically. We have shown that in the strong field regime atom-atom interactions cause the reversible dephasing of Bloch oscillations. As a result, the momentum distribution of the atoms changes periodically from SF to MI-like distributions, with a period given by Eq. (7). Using the original (unscaled) parameters, this period reads
$T_{W}=(d F / W) T_{B}=2 \pi \hbar / W$, where $W$ is the strength of the atom-atom interactions. Since the momentum distribution can be measured easily in the laboratory experiment, this effect suggests an alternative method for studying atom-atom interactions by applying a static force to the system. It is worth to stress one more time that the reported result is valid only in the strong field limit, $d F>J$. If this condition is violated, the evolution operator (6) is no more a diagonal matrix, and the system dynamics get significantly more complicated. An analysis of this latter case will be presented elsewhere.

Discussions with A. Buchleitner are gratefully acknowledged.
[1] F. Bloch, Z. Phys 52, 555 (1928).

[2] C. Zener, Proc. R. Soc. A 145, 523 (1934).

[3] BenDahan et al., Phys. Rev. Lett. 76, 4508 (1996).

[4] S. R. Wilkinson et al., Phys. Rev. Lett. 76, 4512 (1996).

[5] M. G. Raizen, C. Salomon, and Qian Niu, Physics Today 50(8), 30 (1997).

[6] B. P. Anderson and M. A. Kasevich, Science 282, 1686 (1998).

[7] M. Glück, A. R. Kolovsky, and H. J. Korsch, Phys. Rep. 366, 103 (2002).

[8] A. R. Kolovsky, A. V. Ponomarev, and H. J. Korsch, Phys. Rev. A. 66, 053405 (2001).

[9] M. Glück, F. Keck, A. R. Kolovsky, and H. J. Korsch, Phys. Rev. Lett. 86, 3116 (2001); A. R. Kolovsky, and H. J. Korsch, Phys. Rev. A., to appear.

[10] K. Berg-Sorensen and K. Molmer, Phys. Rev. A 58, 1480 (1998).

[11] D. I. Choi and Q. Niu, Phys. Rev. Lett. 82, 2022 (1999).

[12] M. L. Chiofalo, M. Polini, and M. P. Tosi, Eur. Phys. J. D 11, 371 (2000).

[13] O. Morsch et al., Phys. Rev. Lett. 87, 140402 (2001).

[14] H. Fukuyama, R. A. Bari, and H. C. Fogedby, Phys. Rev. B 12, 5579 (1973).

[15] The Wannier states (which should not be confused with Wannier-Stark states) are defined as the Fourier coefficients of the Bloch states over the quasimomentum. For the considered range the $v$, the $l$ th Wannier state is essentially a Gaussian centred at the $l$ th potential well of the optical lattice.

[16] M. P. A. Fisher, P. B. Weichman, G. Grinstein, and
D. S. Fisher, Phys. Rev. B 40, 546 (1989).

[17] S. Sachdev, Quantum phase transitions (Cambridge Univ. Press., Cambridge, 2001).

[18] D. Jaksch, C. Bruder, J. I. Cirac, C. W. Gardiner, and P. Zoller, Phys. Rev. Lett. 81, 3108 (1998).

[19] M. Greiner et al., Nature 415, 39 (2002).

[20] A SF-MI transition can also be tracked by calculating the entropy $S=-\sum_{\mathbf{n}}\left|c_{\mathbf{n}}\right|^{2} \log \left|c_{\mathbf{n}}\right|^{2}$, which changes from $S \sim \log \mathcal{N}(\mathcal{N}$ is the dimension of the Hilbert space) in the SF-phase, to $S \approx 0$ in the MI-phase.

[21] In all numerical simulations periodic boundary conditions were used, i.e., the site $l=L+1$ is identified with $l=1$. Note that in the case $F \neq 0$ this can be done only after elimination of the static term, what is achieved in the interaction representation. Thus, the dynamics of the system (3) was actually calculated on the basis of the time-dependent Hamiltonian $\widetilde{H}(t)=-(J / 2)\left(\exp (-i 2 \pi F t) \sum \hat{a}_{l+1}^{\dagger} \hat{a}_{l}+\right.$ h.c. $)+$ $(W / 2) \sum \hat{n}_{l}\left(\hat{n}_{l}-1\right)$.

[22] One gets exact coincidence between the initial and final distributions only for $W=0$. It might also be useful to mention two obvious facts concerning this case. First, for $W=0$ the momentum distribution $P(k)$ calculated for the many atoms system coincides with that obtained within the single-particle approach. Second, within the framework of our present tight-binding model, the function $P\left(k, t / T_{B}\right)$ does not depend on the particular value of the static force $F$. 